\title{
Infarct size estimation from serial CK MB determinations: peak activity and predictability
}

\author{
J W T FIOLET, H F TER WELLE, F J L VAN CAPELLE, K I LIE
}

From the Department of Cardiology and Clinical Physiology, and the Interuniversity Cardiology Institute, University Hospital, Wilhelmina Gasthuis, Amsterdam, The Netherlands

SUMMARY In 198 patients with acute myocardial infarction serial measurements of plasma creatine kinase isoenzyme $\mathrm{MB}$ (CK $\mathrm{MB}$ ) were performed at four hour intervals. In every patient, maximal CK MB activity (peak activity) was compared with calculated total release per litre plasma. In 28 patients (group 1) sufficient plasma samples were available for calculation of the apparent first order inactivation constant $k_{d}$. Mean apparent $k_{d}$ in group 1 patients was $0.085 \pm 0.018 \mathrm{~h}^{-1}$ (mean $\pm S D$ ). Total release in group 1 was calculated with individual apparent $k_{d}$ values $(Q)$ and with the mean $k_{d}$ value $\left(Q^{\star}\right)$. In the remaining 170 patients (group 2 ), $Q^{\star}$ only was calculated. A linear relation between peak activity $P$ and total release (both $Q$ and $Q^{\star}$ ) was found, extending over the whole range of CK MB peak activities that are routinely observed (4-216 U/l). It was immaterial whether a one or a two compartment model was used: both yielded a close linear relation. Though the mean ratio between $Q^{\star}$ and peak activity depends on the value of $k_{d}$ chosen for calculation of total release (the ratio increasing with increasing $k_{d}$ ), linearity between peak activity and $Q^{\star}$ was found for any value of $k_{d}$ up to $0.4 h^{-1}$.

In group 1 , shapes of calculated $C K M B$ release curves $Q^{\star}(t)$, expressed relative to maximal release $Q(40)$, were sufficiently similar so as to be superimposable; the section of the release curves extending from 12 hours before until two hours after peak time could be tentatively described by a linear time course with a slope of $4 \cdot 2 \pm 0 \cdot 5 \%$ per hour (mean $\pm S D$ ).

We conclude that peak activity of $C K M B$ is a reliable estimate of cumulative $C K M B$ release and may be clinically more practicable than calculation of $Q(40)$. Both the similarity and the large apparently linear section of the calculated enzyme release curves possibly permit early prediction of $\mathrm{Q}(40)$, with acceptable precision.

An enzymatic estimation of the amount of necrotic tissue in myocardial infarction should ideally: (1) be available at an early stage after the onset of ischaemic damage to the tissue, (2) be specific for myocardial tissue, and (3) be obtained easily and reliably. The CK MB isoenzyme meets these criteria reasonably well and is therefore routinely used in our department.

From a theoretical point of view the best enzymatic estimate of infarct size is obtained from complete plasma enzyme activity curves, consisting of many data points of serially obtained blood samples. Both one and two compartment models may be used for calculations, ${ }^{1}{ }^{2}$ depending on the circulatory variables of the particular enzyme used. ${ }^{3}$ It is necessary to continue blood sampling well beyond the time of maximal plasma enzyme activity in order to determine the rate constant of the clearing process for individual patients. ${ }^{3}{ }^{4}$ Good agreement has been shown between CK MB infarct size estimations obtained in this way and histological infarct size determinations at necropsy..$^{5-7}$

Since it is of practical clinical importance to obtain an estimate of infarct size as early as possible, the question is legitimate whether an accurate prediction can be made before the enzyme activity curve is completed. There are two obvious candidates for a similar estimate. The simplest and most frequently adopted procedure is to sample until the peak of the activity curve has been reached, and use the peak value as an estimate of total tissue damage. Published reports indicate that this may be a feasible approach.$^{8-11}$ For CK MB, peak activity is reached about 20 hours after 
onset of infarction. A second, seemingly less gross, procedure is to calculate total release in the same way as is done for complete curves, except that only samples from, for instance, the first $\mathbf{4 0}$ hours are used. Since no individual value of the inactivation constant is available at that time, an approximate value, which is the same for all patients, must be used instead.

The purpose of the present study is to compare total CK MB release, as calculated from the complete plasma enzyme activity curves, both with the release values obtained from curves truncated eight hours after time of maximal activity and with the measured maximal CK MB activity itself. A secondary aim of the study was to explore the possibility of early prediction of total CK MB release based on data points on the ascending limb of the enzyme activity curve. Attempts to do this, based on mathematical algorithms and curve fitting procedures were previously reported as disappointing. ${ }^{11} 12$ Yet we found indications that an acceptable precision may be reached at a time five to 10 hours earlier than the time of maximal activity.

\section{Methods}

Plasma CK MB curves were determined in patients admitted to the coronary care unit with the diagnosis of acute myocardial infarction. Blood samples were drawn four hourly, according to a standard time schedule, the first sample being drawn eight hours after the estimated time of onset of complaints. Sampling continued until at least eight hours after the time of maximal CK MB activity. CK MB plasma activity curves were considered adequate for the study if they contained a minimum of six data points, with at least two points in both the ascending and descending limbs of the curve. One hundred and ninety eight such curves were obtained. In 28 of these (group 1) blood sampling was continued until 40 hours or longer after the onset of infarction. In the remaining 170 patients (group 2) blood sampling was terminated earlier.

Blood samples were drawn in evacuated heparinised tubes and immediately centrifuged twice. After addition of a final concentration of $10 \mathrm{mmol}$ mercaptoethanol the plasma was stored at $-20^{\circ} \mathrm{C}$ until determination (usually within 12 hours). $\mathrm{CK}$ isoenzyme assay and separation were performed as described previously. ${ }^{13}$

For calculation of total CK MB release per litre plasma two mathematical models were applied: (a) the one compartment model, described by

$$
Q(t)=C_{i}(t)+k_{d} \int_{t=0}^{t} C_{i}(t) d t
$$

in which $Q(t)$ is the total activity of CK MB released per litre plasma at time $t, C_{i}(t)$ is plasma $C K M B$ activity observed at time $t$, and $k_{d}$ is the apparent first order inactivation constant; and (b) the two compartment model, described by

$Q(t)=C_{i}(t)+k_{d} \int_{t=0}^{t} C_{i}(t) d t+C_{e}(t)$

in which the additional term $\mathrm{C}_{e}(\mathrm{t})$ allows for the $\mathrm{CK}$ $M B$ activity in the extravascular space. $C_{e}(t)$ is given

$\mathrm{C}_{\mathrm{e}}(\mathrm{t})=\frac{\mathrm{P}}{\mathrm{V}_{\mathrm{i}}} \exp -(\mathrm{P} \times \mathrm{t}) /\left(\mathrm{V}_{\mathrm{e}}\right) \int_{\mathrm{t}=0}^{\mathrm{t}} \mathrm{C}_{\mathrm{i}}(\mathrm{t}) \exp (\mathrm{P} \times \mathrm{t}) /\left(\mathrm{V}_{\mathrm{e}}\right) \mathrm{dt}$

in which $V_{i}$ and $V_{e}$ are the respective intra- and extravascular compartment volumes in litres, and $P$ is the diffusion constant; values for mean $P / V_{i}$ and the mean ratio between $V_{i}$ and $V_{e}$ were taken from a report. ${ }^{3}$ No inactivation is assumed in the extravascular space.

Calculation of $Q(t)$ was performed on a digital PDP 1105 computer. Calculation of the plasma CK MB surface area was based on a cumulative function,

$0.5 C_{n}\left(t_{n}-t_{n-1}\right)+0.5 \sum_{k=1}^{n-1} C_{k}\left(t_{k+1}-t_{k-1}\right)$

Time of first rise of plasma CK MB activity was assumed at $\mathrm{t}=4$ hours, for all patients. ${ }^{13}$ Peak activity of $\mathrm{CK} M B$ refers to measured maximal activity of $\mathrm{CK}$ $M B$ in $\mathrm{U} / 1$.

The symbol $\mathrm{Q}$ in the text refers to $\mathrm{CK} M B$ release calculated with individual values for the first order inactivation constant $k_{d} ; Q^{\star}$ refers to those calculated with mean $k_{d}$ values.

Differences between means were tested by Student's $t$ test. Differences between standard deviations were tested using the $\mathrm{F}$ test. Confidence intervals for correlation coefficients were calculated with Fisher's z-transform.

\section{Results}

TOTAL CK MB RELEASE AND MAXIMAL ACTIVITY In group 1, individual values for the inactivation constants were estimated from semilog plots of at least five data points in the declining limb of the CK MB activity curves. The values of $\mathrm{k}_{\mathrm{d}}$ found were $0 \cdot 085 \pm 0.018$ per hour (mean $\pm S D$ ).

For group 1, total enzyme release was calculated with the one compartment model at $\mathrm{t}=40$ hours with both the mean $k_{d}\left(Q^{\star}(40)\right)$ and with individual $k_{d}$ $(\mathrm{Q}(40))$.

Fig. 1 and 2 show the scattergrams for CK MB peak (1) 


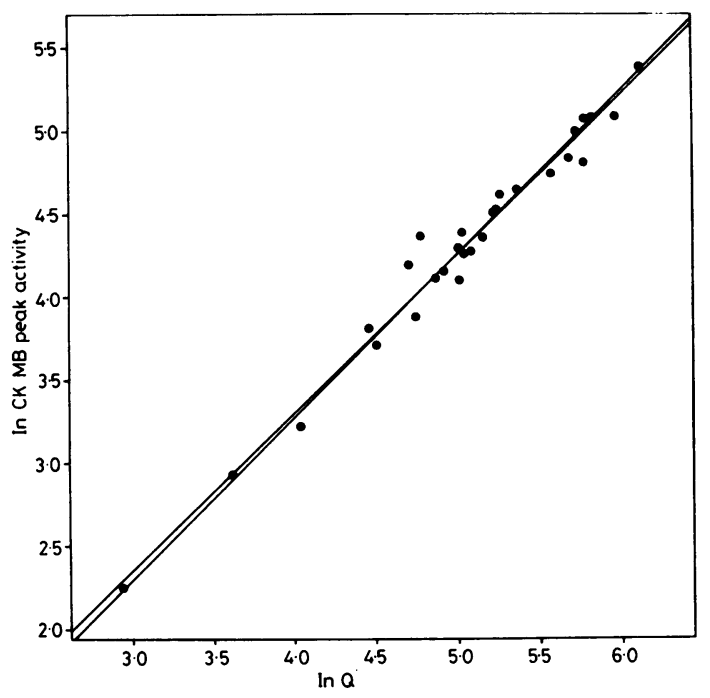

Fig. 1 Linear regression of ln peak activity on total release $\ln Q$ of $C K M B$ in group 1 patients. Total release is calculated at $t=40$ hours, with individual apparent $k_{d}$ values, applying the equation for the one compartment model. Two regression lines are drawn; they correspond with regression of peak activity on ln $Q$ and with regression of $\ln Q$ on peak activity, respectively. The equation of the first line is $y=0.952 x-0.507, r=0.988(95 \%$ confidence interval 0.974-0.995), $S E E=0.109, n=28$.

activity versus $Q$ and $Q^{\star}$ versus $Q$, respectively. In order to meet the distribution requirements for linear regression analysis, data are expressed as the natural logarithm of enzyme activities. The correlation coefficients for both regressions are high. Comparison of the standard errors of estimate of the regressions in Fig. 1 and 2 shows that peak activity in group 1 does not indicate a less precise estimate of $Q(40)$ than does $Q^{\star}(40)$. In fact, the correlation coefficient for peak activity is significantly greater than that for $Q^{\star}(40)$ $(p<0.05)$. It follows that the ratio between total release and measured peak activity is essentially constant over a wide range of CK MB activities (up to at least approximately $220 \mathrm{U} / 1$ ).

The mean ratio between $Q(40)$ and peak activity in individual patients was $2 \cdot 12 \pm 0 \cdot 23$ (mean $\pm S D$ ).

In order to determine whether the values for variables characterising the shape of the plasma CK MB activity curve show statistically significant differences

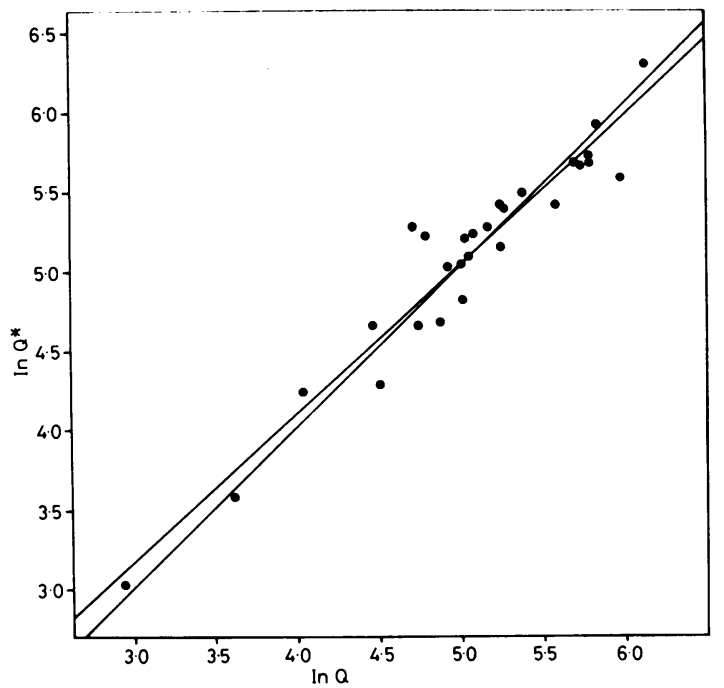

Fig. 2 Linear regression of $\ln Q^{\star}$ on $\ln Q$ of $C K M B$ in group 1 patients. Both $Q^{\star}$ and $Q$ are calculated at $t=40 \mathrm{~h}$, applying the one compartment model; $Q^{\star}$ is calculated with mean apparent $k_{d}=0.085 h^{-1}$, and $Q$ with individual apparent $k_{d}$ values. Linear regression equation is $y=0.938 x+0.359, r=0.962(95 \%$ confidence interval 0.918-0.982), $S E E=0.194, n=28$.

between patients with high and low values for the $\mathrm{Q}(40) /$ peak ratio, we divided group 1 patients into two subgroups having ratio values greater or smaller than $2 \cdot 12$. The results are shown in Table 1 . No statistically significant differences existed in peak activity and peak time, but $k_{d}$ values were significantly different $(p<0.05)$ between the two subgroups.

Fig. 3 shows the correlation between peak activity and $Q^{\star}(t)$ in 170 patients of group 2 . $Q^{\star}(t)$ was calculated, on the average, approximately eight hours after peak time. The mean ratio between $Q^{\star}(t)$ and peak activity is $2.05 \pm 0.23$ (mean $\pm \mathrm{SD}$ ), somewhat lower than that seen in Fig. 1. The difference is not statistically significant.

In group 1 we also calculated $Q(40)$, using the two compartment model. As in the one compartment model, the relation between peak activity and $Q(40)$ is linear (not shown), $r=0.99$. The mean ratio of $Q(40)$ to peak activity in this case is $2.33 \pm 0.26$ (mean $\pm S D$ ).

Table 1 Characteristics of plasma CK MB curves in patients with high and low ratios of release/peak

\begin{tabular}{lllll}
\hline & $\begin{array}{l}\text { Ratio } \\
\text { (Mean } \pm S D)\end{array}$ & $\begin{array}{l}\text { CKMB } \\
\text { Peak activity } \\
\text { (U/l) }\end{array}$ & $\begin{array}{l}\text { Peak time } \\
(h)\end{array}$ & $\begin{array}{l}k_{d} \\
\left(h^{-1}\right)\end{array}$ \\
\hline $\begin{array}{l}\text { Ratio } \leqslant 2 \cdot 12 \\
\mathrm{n}=14\end{array}$ & $1 \cdot 96 \pm 0 \cdot 17$ & $91 \pm 55$ & $22 \cdot 0 \pm 3 \cdot 0$ & $0.077 \pm 0.016$ \\
$\begin{array}{l}\text { Ratio }>2.12 \\
\mathrm{n}=14\end{array}$ & $2 \cdot 29 \pm 0.14$ & $86 \pm 43$ & $21 \cdot 6 \pm 3 \cdot 0$ & $0.092 \pm 0.017$ \\
\hline
\end{tabular}




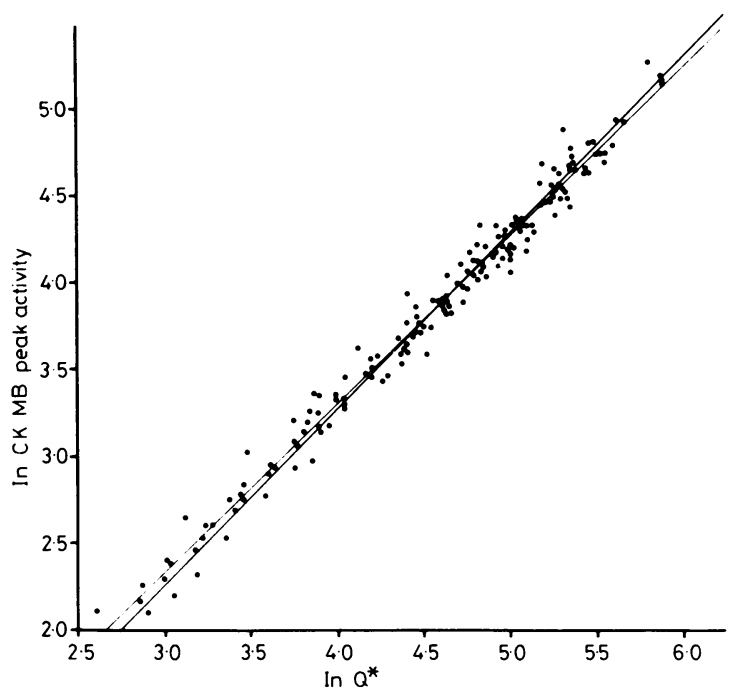

Fig. 3 Linear regression of $\ln$ peak activity on $\ln Q^{\star}$ of $C K$ $M B$ in group 2 patients. $Q^{\star}$ is calculated as for Fig. 2 , at time $t=8 h$ after peak time. Linear regression equation is $y=0.973 x-0.587, r=0.991$ ( $95 \%$ confidence interval $0.988-0.995), S E E=0.099, n=170$.

That this is higher than the ratio found in the one compartment model is because of the term for the extravascular CK MB contribution to $Q(40)$ in formula 2. Table 2 shows the ratio between $Q(40)$ values calculated by applying both the one and the two compartment models. $\mathrm{Q}(40)$ values in the two compartment model are approximately $12 \%$ higher than those in the one compartment model. When a high mean value for $k_{d}$ (see below) is used for calculating $Q^{\star}(40)$, the difference between one compartment $Q^{\star}(40)$ and two compartment $Q^{\star}(40)$ values becomes even less.

\section{INFLUENCE OF INACTIVATION RATE CONSTANT}

$Q(t)$, according to formulae 1 and 2, largely depends on the value of $k_{d}$. Recent reports indicate that $k_{d}$ estimated from semilog plots underestimates the true elimination constant by a factor two to four. Fig. 4 shows the effect of using a high $k_{d}$ value on the ratio of $Q(t)$ to peak activity as compared with using the apparent individual $k_{d}$ value in an individual patient. Using the high $k_{d}$ value in calculating $Q(t)$ has two effects: firstly, the ratio $Q(40)$ to peak activity increases, and, secondly, enzyme release appears to

Table 2 Ratio of $C K M B$ release $Q$ in two compartment model and in one compartment model; dependence on value of $k_{d}$

\begin{tabular}{ll}
\hline & $\begin{array}{l}\text { Ratio } \\
(\text { Mean } \pm S D)\end{array}$ \\
\hline Individual apparent $\mathrm{k}_{\mathrm{d}}$ & $1 \cdot 118 \pm 0.015$ \\
$\mathrm{k}_{\mathrm{d}}=0.250 \mathrm{~h}^{-1}$ & $1.044 \pm 0.002$ \\
\hline
\end{tabular}

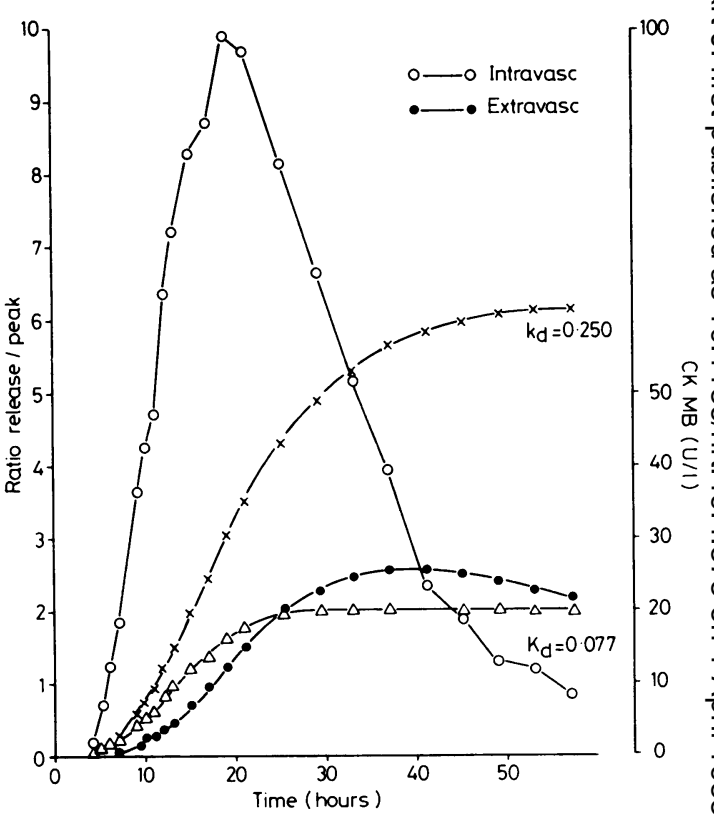

Fig. 4 Time course of ratio between $Q(t)$ and peak activity in an individual patient. $\bigcirc-O$ time course of intravascular activity of $C K M B C_{i}(t)$; $\longrightarrow$ time course of calculated extravascular activity $C_{e}(t) ; \triangle \longrightarrow \triangle$ ratio between $Q(t)$ and peak activity, $k_{d}=0.077 h^{-1} ; \times \longrightarrow \times$ ratio between $Q^{\star}(t)$ and peak activity, $k_{d}=0.250 h^{-1} . Q$ and $Q^{\star}$ are calculated by applying the two compartment model.

continue for a much longer period of time. In this particular patient, enzyme release at $k_{d}=0.077$ seems -

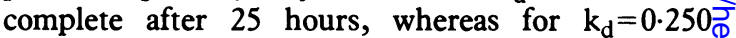
enzyme release continues until approximately $50 \stackrel{\mathrm{g}}{7}$ hours.

When high $k_{d}$ values are used for $Q^{\star}(40)$ calcula $\frac{3}{2}$. tion, linearity between peak activity and $\mathrm{Q}^{\star}(40)$ iso̊ maintained. The ratio of $Q^{\star}(40)$ to peak activity, however, increases with increasing $k_{d}$. This is illus-o trated in Fig. 5. For all $k_{d}$ values, essentially constant ratios are found, with similar coefficients of variation. 으․

PREDICTABILITY OF TOTAL CK MB RELEASE

G

As illustrated above, measured maximal $\mathrm{CK} M \mathrm{MB}^{\mathrm{N}}$ activity is a reasonably good predictor of total $C K M B N$ release. Acceptable prediction may well be possibleo earlier than time of peak activity, since neither peako activity itself nor the time of its occurrence has a specific significance in the release curve.

Normalised release curves of the patients in group 10 are shown on a relative time scale in Fig. 6. Zero time is arbitrarily set at $50 \%$ of enzyme release, and all $Q^{\star}(t)$ values of the individual patients are calculated市 relative to $Q^{\star}$ at $t=40$ hours. $Q^{\star}(t)$ was calculatedo using the two compartment model with $\mathrm{k}_{\mathrm{d}}=0.250$. 


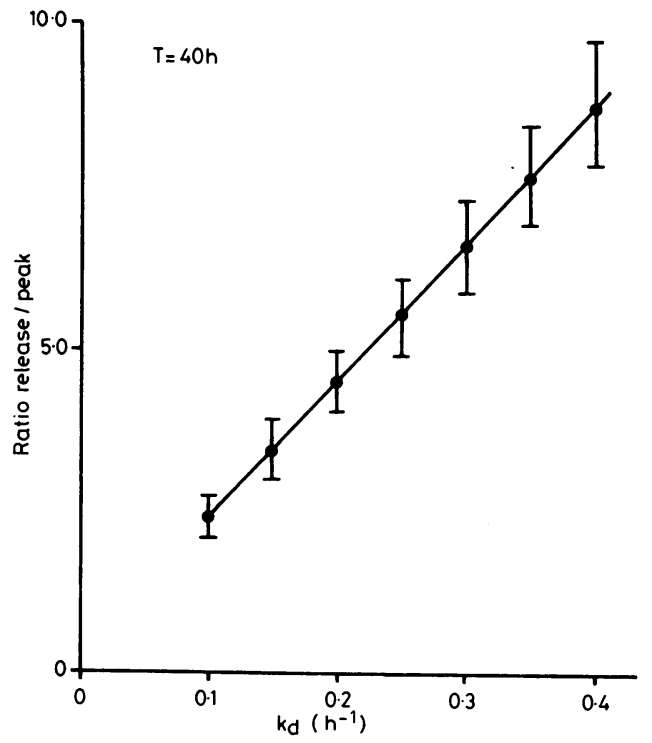

Fig. 5 Dependence of the ratio of $Q^{\star}(t)$ and peak activity on the value of $k_{d}$. $Q^{\star}$ is calculated at $t=40 \mathrm{~h}$ for group 1 patients. Standard errors are indicated by bars.
The release curves of all 28 patients of group 1 appear superimposable, though not identical. A large part of the curve in Fig. 6 may be tentatively described as a linear time course from about minus 10 hours to plus four hours, with a slope of approximately $4.2 \%$ per hour. From this mean slope, prediction of $Q^{\star}(40)$ may be possible from multiple data points in the early rising section of the plasma $\mathrm{CK} M B$ activity curve. The predictive precision depends on the interindividual variation of the slopes in the linear part of the $Q(t)$ curves. These slopes are $4 \cdot 20 \pm 0.45 \%$ (mean $\pm S D)$. Mean peak time relative to arbitrary dictive precision of about $10 \%$.

For patients in group 1, the mean peak time relative to the time of onset of complaints was $21 \cdot 8 \pm 3.0$ hours $($ mean $\pm S D)$. Mean peak time relative to arbitrary zero time (time at which $Q(t)=50 \%$ ) was $1.7 \pm 1.4$ hours (mean $\pm \mathrm{SD}$ ). This $\mathrm{SD}$ is significantly smaller $(p<0.005)$ than the one referred to first.

\section{Discussion}

In previous studies, cumulative enzyme release was related to infarct size, mainly by using curves obtained from serial CK measurements. ${ }^{1} 2$ 14-19 Infarct size estimates from these curves have shown a

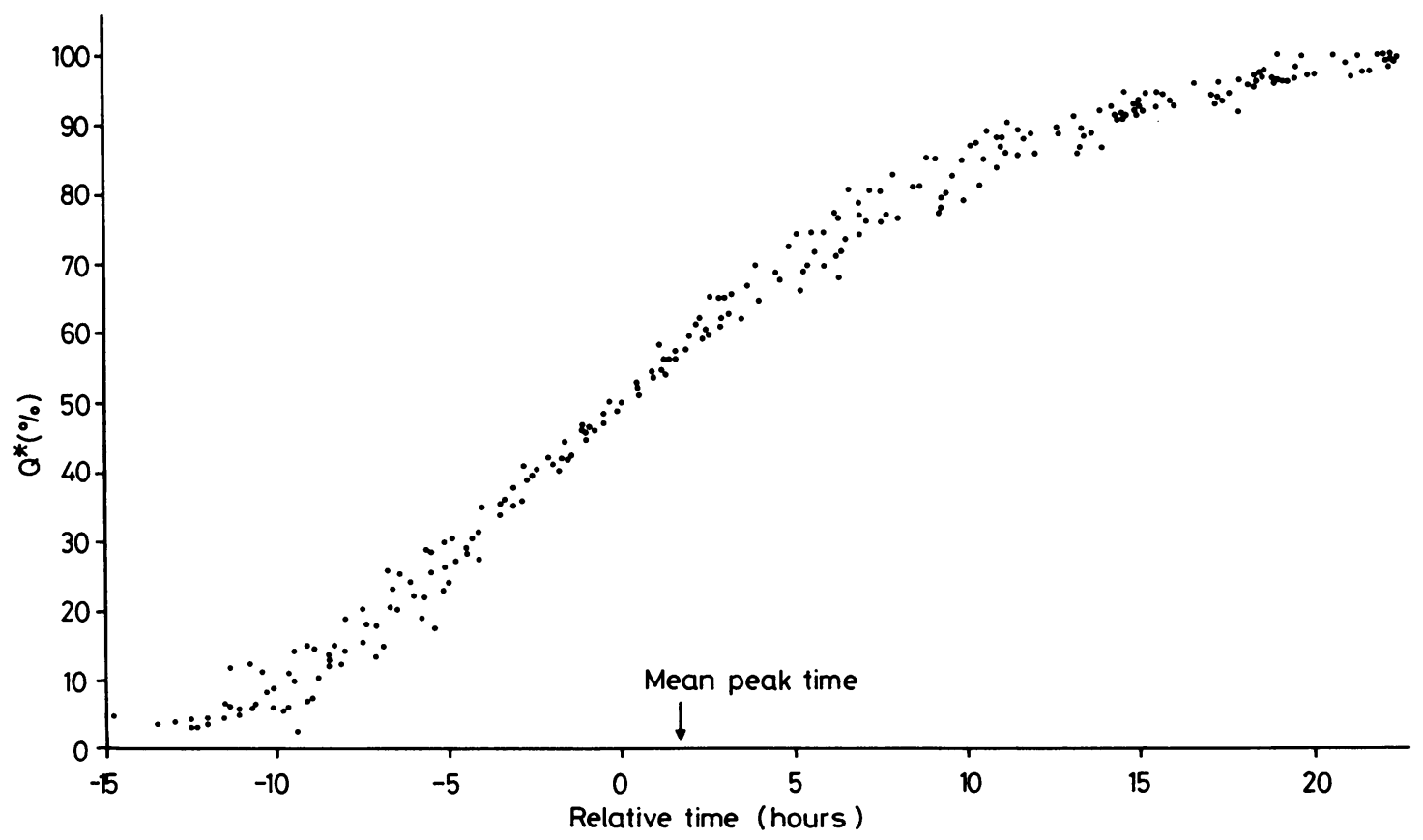

Fig. 6 Normalised release curves of $C K M B$ in group 1 patients. Every individual release curve $Q^{\star}(t)$ is normalised relative to the individual $Q$ at $t=40 \mathrm{~h}$. The individual release curves are plotted on a relative time scale, which is arbitrarily set to zero at $Q^{\star}=50 \%$. Mean time of maximal plasma CK MB activity is indicated by the arrow. $Q^{\star}(t)$ is calculated by applying the two compartment model, with $k_{d}=0 \cdot 250 h^{-1}$. 
relation to mortality and left ventricular performance. ${ }^{8} 11141617$ Though it has been stressed that estimates of infarct size from cumulated CK release should be interpreted with caution, ${ }^{199}$ good correlation with postmortem infarct size determinations was shown. 19

Because of the high cardiospecificity of the CK MB isoenzyme, its cumulated release after myocardial infarction should, theoretically, give more accurate estimates of infarct size, especially under circumstances where less specific enzymes could contribute activity from non-cardiac origin. ${ }^{20}$ In patients with uncomplicated acute myocardial infarction, similar shapes of CK MB curves were found, irrespective of whether or not these patients received intramuscular injections. The shapes of their total CK curves, however, differed. ${ }^{20} \mathrm{~A}$ highly significant correlation was reported recently between CK MB estimated infarct size and postmortem determinations. ${ }^{6} 7$ In addition, correlation was found with radionuclide estimates of infarct size. ${ }^{21}$

This study shows that measured maximal plasma CK MB activity in patients with acute myocardial infarction is linearly correlated with CK MB release per litre plasma, accumulated during 40 hours after the onset of symptoms $(Q(40))$. This linear relation holds over the whole range of maximal CK MB activities found in patients with acute myocardial infarction. Thus, an average ratio between measured maximal activity and calculated cumulative release exists. The value of this ratio is scarcely influenced by the kind of model used in the calculations. This is in agreement with earlier findings, especially for enzymes with relatively high $k_{d}$ values. ${ }^{34}$

Apparently, the value of the ratio between $Q(40)$ and maximal activity is highly dependent on the value of $k_{d}$ used in calculating $Q(40)$. On the basis of the fundamental similarity of release kinetics for different enzymes from infarcting myocardium, it has been argued that true $k_{d}$ values for CK $M B$ are considerably larger than those determined from the apparent monoexponential declining limb of the $\mathrm{CK}$ MB curve and, in fact, cannot be determined in that manner. ${ }^{3} 4$ This is also in agreement with high $k_{d}$ values for CK and its isoenzymes found in experimental studies involving intravenous injection of the isoenzymes in the dog. ${ }^{22}{ }^{23}$ For CK MB injected into baboons, however, a much lower value was reported. ${ }^{24}$

The consequence of a much higher $k_{d}$ value is that enzyme release may continue beyond $\mathbf{4 0}$ hours after the onset of complaints (cf Fig. 4). Therefore, $Q(40)$ values calculated with high $k_{d}$ will underestimate the true total CK MB released per litre plasma and thus somewhat underestimate the true value of the ratio between $Q$ and maximal CK MB activity.

As shown in Figs. 1 to 3, strong correlations bet- ween peak activity and cumulated release were found, both when an individual and a mean value was used in $\mathrm{c}$ the calculation of cumulated release. From Table $1 \mathrm{it} \Rightarrow$ follows, however, that lower ratios of $\mathrm{Q} /$ peak are $\frac{\text { P }}{?}$ associated with lower values for $k_{d}$. Thus, using a? mean $k_{d}$ value in calculating $Q$ for an individual $\frac{}{\bar{N}}$ patient introduces an error proportional to the interindividual variation of $k_{d}$. The coefficient of vari- $Q$ ation found for the $Q /$ peak ratio was approximatelyos $11 \%$, irrespective of the mean value of $k_{d}$ used(Fig. 5). A much larger variation than $11 \%$ wasreported in true $k_{d}$ values for CK MB. ${ }^{4}$ It must bew noted, however, that true $k_{d}$ values, because of thes fundamental impossibility of determining them directly, have been derived indirectly by comparinget the release kinetics of different enzymes.

Several other factors contribute to the interin- $\omega$ dividual variation in the $Q(40) /$ peak ratio. Firstly, the ${ }^{\omega}$ $5 \%$ coefficient of variation of the $\mathrm{CK} \mathrm{MB}$ assay ${ }^{13}$ con- 5 tributes fully to the variability of the peak activity and partially to the variability of $Q(40)$. Secondly, theo underestimation of the true maximal activity by measured maximal activity is an additional source of $\vec{\varnothing}$ variation. For sampling intervals of four hours, it wasc recently found that measured maximal activity of $\mathrm{CK}$ D deviates from true peak activity by less than $6 \%$ on the average; CK values remained at greater than $90 \%$ of peak for a mean of 7.6 hours. ${ }^{11}$ Thirdly, accuracy and precision of the calculation of $Q(40)$ depend on the sampling frequency. For CK $M B$ it has been shown that cumulative release calculated for sampling inter-응 vals of one hour correlated significantly with that cal-culated for eight hour sampling intervals $(r=0.997$, $\mathrm{SEE}=6 \%^{5}$ ). Furthermore, for our calculations it was

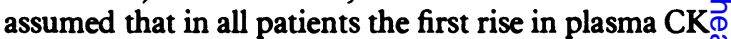
MB activity occurred four hours after the onset of complaints. This first activity increase, however, may? have occurred earlier, especially in patients with. larger infarctions. Moreover, there is an intrinsico uncertainty in the assumed time of onset of com- $₹$ plaints. A fourth factor which may contribute to the? observed variation in the ratio $Q(40) /$ peak is the poss- $\rightarrow$ ible occurrence of small infarct extension or expan-․ㅡ. sion. ${ }^{18} 25$ This cannot be ruled out because selection of patients for this study was based only on the clinical diagnosis of acute myocardial infarction upon admission, and on the criteria for suitable CK MB plasmacis activity curves, mentioned in the method section? Clear, double-peaked plasma activity curves, how ever, were not observed. Alternatively, statistically significant deviation from the mean of the $Q(40) /$ peak: value for a particular patient may be used to establish ${ }^{0}$ or confirm the diagnosis of reinfarction.

Our results indicate that CK MB peak activity is reliable estimate of ultimate total enzyme release and $\stackrel{\mathscr{\Omega}}{\varrho}$ therefore, that this release is predictable. Prediction 
occurs at peak time, which is considerably earlier than the time at which enzyme release is completed. There is no reason why predictions could not be made even earlier, since peak time has no special significance in the time course of enzyme release. Successful prediction of infarct size was reported from data points in the first seven hours after initial CK rise in patients with acute myocardial infarction. ${ }^{15}$ Other attempts at prediction have been disappointing thus far; variable and unacceptable inaccuracy was shown, especially early in the time course. ${ }^{11} 12$ This inaccuracy, however, may be the result of the predictive procedure used, which involves curve-fitting to successively fewer data points. This procedure may introduce considerable uncertainties in the coefficients of the "fitting" equation, especially if only a few early data points are available.

The results described in Fig. 6 indicate a possibly more promising approach. Superimposed release curves appear similar and linear for a considerable period of time. Enzyme release curves of individual patients show no appreciable difference in slope of the linear sections. The mean slope gives the fraction of total enzyme released per unit time. Whether or not the linear section of the release curve $\left(Q^{\star}(t)\right)$ has been reached can be evaluated from CK MB activity in multiple, early, densely-spaced plasma samples. In this way, the difficulties encountered in determining the exact time of onset of complaints are avoided. Subsequently, ultimate $Q \star(40)$ may be predicted from both the early $Q^{\star}(t)$ values in the linear section of the release curve and the above-mentioned mean slope value. This approach may eliminate possible errors caused by curve fitting.

The feasibility and predictive precision of this procedure are presently under investigation. A predictive imprecision of about $10 \%$ (coefficient of variation of individual slopes) seems attainable, perhaps even less if precision of the CK MB assay, especially at low activities, can be improved. Clinical interventions may reduce infarct size by about $20 \% .^{21} 26$ Such interventions may be evaluated in the individual patient if a predictive imprecision of less than $10 \%$ can be achieved.

We gratefully acknowledge the critical assistance of Ms S J A Tasseron in the preparation of the manuscript.

\section{References}

1 Shell WE, Kjekshus JK, Sobel BE. Quantitative assessment of the extent of myocardial infarction in the conscious dog by means of analysis of serial changes in serum creatine phosphokinase activity. $\mathcal{f}$ Clin Invest 1971; 50: 2614-25.
2 Witteveen SAGJ, Hemker HC, Hollaar L, Hermens WT. Quantification of infarct size in man by means of plasma enzyme levels. Br Heart $\mathcal{F}$ 1975; 37: 795-803.

3 Willems GM, Muijtiens AMM, Lambi FHH, Hermens WT. Estimation of circulatory parameters in patients with acute myocardial infarction. Significance for calculation of enzymatic infarct size. Cardiovasc Res 1979; 13: 578-87.

4 Willems GM, Visser MP, Krill MTA, Hermens WT. Quantitative analysis of plasma enzyme levels based upon simultaneous determination of different enzymes. Cardiovasc Res 1982; 16: 120-31.

5 Grande P, Naestoft J, Christiansen C. An easy and reliable estimation of acute myocardial infarct size from serum CK MB measurements. Eur $\mathcal{F}$ Cardiol 1980; 11: 71-7.

6 Grande P, Fischer-Hansen B, Christiansen C, Naestoft J. Acute myocardial infarct size estimated by serum CK MB determinations: clinical accuracy and prognostic relevance utilizing a practical modification of the isoenzyme approach. Am Heart f 1981; 101: 582-6.

7 Grande P, Fischer-Hansen B, Christiansen C, Naestoft J. Estimation of acute myocardial infarct size in man by serum CK MB measurements. Circulation 1982; 65: 756-64.

8 Kahn JC, Gueret P, Baudet M, Rocha P, Bardet J, Bourdarias JP. Clinical assessment of infarct size by serial determinations of serum creatine phosphokinase activity. Eur $\mathcal{F}$ Cardiol 1979; 9: 21-37.

9 Chapelle JP, Albert A, Heusghem C, Smeets JP, Kulbertus HE. Predictive value of serum enzyme determinations in acute myocardial infarction. Clin Chim Acta 1980; 106: 29-38.

10 van der Laarse A, Davids HA, Hollaar L, van der Valk EJM, Witteveen SAGJ, Hermens WT. Recognition and quantification of myocardial injury by means of plasma enzyme and isoenzyme activities after cardiac surgery. $\mathrm{Br}$ Heart $\mathcal{F}$ 1979; 41: 660-7.

11 Ryan W, Karliner JS, Gilpin EA, Covell JW, De Luca M, Ross J Jr. The creatine kinase curve area and peak creatine kinase after acute myocardial infarction: usefulness and limitations. Am Heart $\mathcal{f}$ 1981; 101: 162-8.

12 Danner SA, Smit EM, Hart GAM, Oosting H, Dunning AJ. Prediction of infarct size from serial CK determinations: evaluation by clinical studies and computer simulation. Clin Chim Acta 1979; 97: 45-7.

13 Fiolet JWT, Willebrands AF, Lie KI, ter Welle HF. Determination of creatine kinase isoenzyme MB (CK MB): comparison of methods and clinical evaluation. Clin Chim Acta 1977; 80: 23-35.

14 Sobel BE, Bresnahan GR, Shell WE, Yoder RD. Estimation of infarct size in man and its relation to prognosis. Circulation 1972; 46: 640-8.

15 Shell WE, Lavelle JF, Covell JW, Sobel BE. Early estimation of myocardial damage in conscious dogs and patients with evolving acute myocardial infarction. f Clin Invest 1973; 52: 2579-90.

16 Mathey D, Bleifeld W, Hanrath P, Effert S. Attempt to quantitate relation between cardiac function and infarct size in acute myocardial infarction. Br Heart $\mathcal{F} 1974 ; 36$ : 271-9.

17 Mathey D, Bleifeld W, Buss H, Hanrath P. Creatine 
kinase release in acute myocardial infarction: correlation with clinical, electrocardiographic, and pathological findings. Br Heart $\mathcal{F}$ 1975; 37: 1161-8.

18 Norris RM, Whitlock RML, Barratt-Boyes C, Small CW. Clinical measurement of myocardial infarct size. Modification of a method for the estimation of total creatine phosphokinase release after myocardial infarction. Circulation 1975; 51: 614-20.

19 Bleifeld W, Mathey D, Hanrath P, Buss H, Effert S. Infarct size estimated from serial serum creatine phosphokinase in relation to left ventricular hemodynamics. Circulation 1977; 55: 303-11.

$19^{9}$ Roe CR. Validity of estimating myocardial infarct size from serial measurements of enzyme activity in the serum. Clin Chem 1977; 23: 1807-12.

20 Sobel BE, Roberts R, Larson KB. Estimation of infarct size from serum MB creatine phosphokinase activity: applications and limitations. Am $\mathcal{f}$ Cardiol 1976; 37: 474-85.

21 Morrison J, Reduto L, Pizzarello R, Geller K, Maley T, Gulotta S. Modification of myocardial injury in man by corticosteroid administration. Circulation 1976; 53 (suppl 1): 200-3.
22 Rapaport E. The fractional disappearance rate of separate isoenzymes of creatine phosphokinase in the dog. Cardiovasc Res 1975; 9: 473-7.

23 Roberts R, Sobel BE. Effect of selected drugs and myocardial infarction on the disappearance of creatine kinase from the circulation in conscious dogs. Cardiovasc Res 1977; 11: 103-12.

24 Yasmineh WG, Pyle RB, Nicoloff DM. Rate of decay and distribution volume of $\mathrm{MB}$ isoenzymes of creatine kinase, intravenously injected into the baboon. Clin Chem 1976; 22: 1095-7.

25 Hutchins GM, Bulkley BH. Infarct expansion versus extension: two different complications of acute myocardial infarction. Am $\mathcal{Y}$ Cardiol 1978; 41: 1127-32.

26 Shell WE, Sobel BE. Biochemical markers of ischemic injury. Circulation 1976; 53 (suppl 1): 98-106.

Requests for reprints to Dr J W T Fiolet, Department of Cardiology and Clinical Physiology, University Hospital, Wilhelmina Gasthuis, Eerste Helmersstraat 104, 1054 EG Amsterdam, The Netherlands. 